\title{
Theory of amplified dispersive Fourier transformation
}

\author{
Keisuke Goda, ${ }^{1}$ Daniel R. Solli, ${ }^{1}$ Kevin K. Tsia, ${ }^{2}$ and Bahram Jalali ${ }^{1}$ \\ ${ }^{1}$ Department of Electrical Engineering, University of California, Los Angeles, California 90095, USA \\ ${ }^{2}$ Deparment of Electrical and Electronic Engineering, University of Hong Kong, Pokfulam Road, Hong Kong
}

(Received 29 June 2009; published 19 October 2009)

\begin{abstract}
Amplified dispersive Fourier transformation (ADFT) is a powerful technique that maps the spectrum of an optical pulse into a time-domain waveform using group-velocity dispersion (GVD) and simultaneously amplifies it in the optical domain. It replaces a diffraction grating and detector array with a dispersive fiber and single photodetector, greatly simplifying the system and, more importantly, enabling ultrafast real-time spectroscopic measurements. Here we present a theory of ADFT by deriving the general equation and spectral resolution for ADFT and studying the evolution of the pulse spectrum into time, the effect of GVD coefficients on ADFT, and the requirement for dispersion. This theory is expected to lend valuable insights into the process and implementation of ADFT.
\end{abstract}

DOI: 10.1103/PhysRevA.80.043821

PACS number(s): 42.62.Fi, 42.65.Re

\section{INTRODUCTION}

Spectroscopy is a ubiquitous diagnostic technique that identifies a solid, liquid, or gas based on its chemical and physical (molecular or vibrational) signature. It is a tool that has created entirely new fields in science such as modern physics via the discovery of blackbody radiation and atomic spectra, chemistry by identifying chemical elements and compounds, biology and medicine via fluorescence and Raman spectroscopy, and astronomy by detecting absorption and emission lines from distant stars and galaxies. Surprisingly, at the heart of today's spectrometers is the diffraction grating invented by Fraunhofer in 1814 and later used by Kirchoff and Bunsen in 1859 to demonstrate that different elements and compounds have unique signatures.

Fast real-time spectroscopy is an extremely challenging type of measurement; yet, it is needed for studying fast dynamic processes such as those occurring in chemical reactions, molecular transformations, and other transient phenomena. Unfortunately, conventional grating-based spectrometers are often too slow because they either employ moving components (e.g., a rotating grating or translating slit) or rely on a detector array [e.g., a charge-coupled device (CCD)] with limited frame rate (typically less than $\mathrm{kHz}$ ). Also, there is the fundamental tradeoff between sensitivity and speed; at high scan rates, fewer photons are detected during each scan. Therefore, traditional spectrometers are not ideal for ultrafast real-time measurements because there is no means to overcome this limitation other than using highpower excitation sources-an approach that can damage or modify the specimen under test, especially for biological samples.

Amplified dispersive Fourier transformation (ADFT) $[1-5]$ is a relatively new and potentially powerful technique for overcoming the limitations of the traditional optical spectrometers. ADFT is an optical process that exploits the analogy between paraxial diffraction and temporal (group velocity) dispersion, and overcomes the loss inherent in a dispersive medium using optical amplification. It maps the spectrum of an optical pulse into a temporal waveform using group-velocity dispersion (GVD) and simultaneously ampli- fies it in the optical domain. ADFT replaces the spatial disperser (e.g., the diffraction grating) and detector array (e.g., the CCD) in conventional spectrometers with a GVD device (e.g., a dispersive optical fiber) and single photodetector, greatly simplifying the system and, more importantly, enabling ultrafast real-time spectroscopic measurements. Originating from the work on photonic time-stretch analog-todigital conversion [6], ADFT allows the optical spectrum to be measured directly in time. By sampling the temporal waveform, a real-time analog-to-digital converter or a digital oscilloscope effectively samples the optical spectrum at ultrahigh scan rates, significantly beyond what is possible with conventional grating-based spectrometers. With distributed optical amplification in the dispersive fiber, ADFT overcomes the fundamental tradeoff between loss and dispersion, hence circumventing the tradeoff between sensitivity and speed. ADFT has been used for numerous applications, starting with the time-stretch analog-to-digital converter [6], absorption [5] and Raman [2] spectroscopy, optical frequencydomain reflectometry [3], displacement sensing and barcode reading [4], and microscopy [1].

In ADFT, the desirable features for a GVD device are high total dispersion, low loss, large optical bandwidth, and constant dispersion over the bandwidth of interest. At fiberoptic communication wavelengths, the dispersion compensation fiber (DCF) offers an optimum combination of these parameters and has been used in many applications [1-5,7-12]. There are also fibers for shorter wavelengths (e.g., $800 \mathrm{~nm}$ ) that provide adequate dispersion although with much higher loss-to-dispersion ratio than the DCF. While the loss in the DCF can also be compensated by discrete optical amplifiers such as erbium-doped fiber amplifiers (EDFAs) or even semiconductor optical amplifiers (SOAs), distributed Raman amplification within the dispersive fiber is superior because it maintains a relatively constant signal level throughout the ADFT process. This important property maximizes the signal-to-noise-and-distortion ratio by keeping the signal power away from low power (noisy) and high power (nonlinear) regimes. This advantage of distributed Raman amplification over the use discrete amplifiers is known in long haul fiber-optic communication links [13]. In addition to a lower noise figure, the intrinsic properties of Raman 


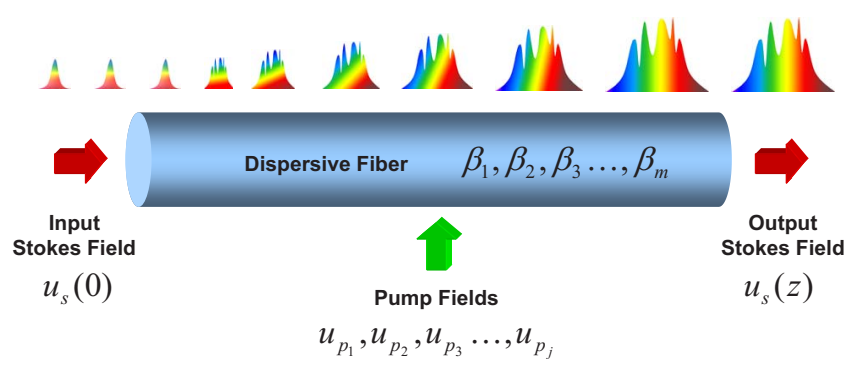

FIG. 1. (Color online) Amplified dispersive Fourier transformation. $u_{S}(0)$ and $u_{S}(z)$ are the normalized amplitude of the input and output Stokes fields, respectively, $z$ is the propagation distance (equivalent to the dispersion length), $\beta_{m}$ are the GVD coefficients at the Stokes frequency, and $u_{p_{j}}$ is the normalized amplitude of pump field $j$. In the ADFT, the spectrum of each pulse is mapped into a time-domain waveform and simultaneously amplified by distributed Raman amplification

amplification are also favorable for ADFT, such as its widely tunable gain spectrum and its naturally broadband gain spectrum, which is naturally broadband due to the amorphous nature of optical glass fibers. The gain bandwidth can be further broadened by using multiwavelength pump lasers $[1,5]$. Also, extremely broadband gain spectra can be realized using incoherent pump sources [2]. Raman-amplified dispersive elements also eliminate the need for high-power optical sources, which can potentially damage or modify the sample under study as mentioned above [1].

In this paper, we present a theory of ADFT. This is motivated by the recent successful applications of ADFT in spectroscopy $[2,5]$ and imaging $[1,3,4]$ that require a quantitative understanding of the ADFT process including its spectral resolution and dependence on linear and nonlinear GVD. We first derive the central equation for ADFT from the generalized nonlinear Schrödinger equation with all GVD coefficients in the presence of multiple pump fields (Sec. II). We also derive the physical spectral resolution set by the frequency-to-time transformation as well as the digital spectral resolution set by the bandwidth of the back-end analogto-digital converter or digitizer that captures the temporal waveform. To analyze the accuracy of ADFT, we then examine the evolution of the pulse spectrum into a time-domain waveform, the effect of linear and nonlinear GVD, and the minimum GVD required to reach the temporal equivalent of the stationary-phase approximation-the mathematical condition for ADFT (Sec. III).

\section{EQUATION FOR AMPLIFIED DISPERSIVE FOURIER TRANSFORMATION}

In this section, starting with the generalized nonlinear Schrödinger equation with all the GVD coefficients and multiple pump fields (derived in Appendix A), we solve the equation analytically under some basic assumptions to obtain the ADFT equation, which is then applied in a few examples. We also investigate the requirement for the total amount of dispersion necessary to perform the stationary-phase approximation that enables the ADFT in each case. Eventually, we derive the spectral resolution determined by the ADFT.

Figure 1 shows the generalized schematic of the ADFT. We start with the generalized nonlinear Schrödinger equation with all the GVD coefficients and multiple pump fields [Eq. (A19) derived in Appendix A] for the normalized Stokes field amplitude $u_{S}$,

$$
\begin{aligned}
& \frac{\partial u_{S}}{\partial z}-i \sum_{m=1}^{\infty} \frac{i^{m} \beta_{m}}{m !} \frac{\partial^{m} u_{S}}{\partial t^{m}}+\frac{\alpha_{S}}{2} u_{S} \\
& =i \gamma_{S}\left(1-f_{R}\right) u_{S}\left[\left|u_{S}(t)\right|^{2}+2 \sum_{j=1}^{\infty}\left|u_{p_{j}}(t)\right|^{2}\right] \\
& \quad+i \gamma_{S} f_{R} u_{S} \int_{-\infty}^{\infty} h_{R}\left(t-t^{\prime}\right)\left[\left|u_{S}\left(t^{\prime}\right)\right|^{2}+\sum_{j=1}^{\infty}\left|u_{p_{j}}\left(t^{\prime}\right)\right|^{2}\right] d t^{\prime} \\
& \quad+i \gamma_{S} f_{R} \sum_{j=1}^{\infty} u_{p_{j}} \int_{-\infty}^{\infty} h_{R}\left(t-t^{\prime}\right) u_{S}\left(t^{\prime}\right) u_{p_{j}}^{*}\left(t^{\prime}\right) \\
& \quad \times e^{-i\left(\omega_{p_{j}}{ }^{-\omega_{S}}\right)\left(t-t^{\prime}\right)} d t^{\prime},
\end{aligned}
$$

where $z$ is the propagation distance (equivalent to the dispersion length), $\beta_{m}$ are the GVD coefficients for the Stokes field, $\gamma_{S}$ and $\alpha_{S}$ are the nonlinear and loss parameters for the Stokes field, respectively, $u_{p_{j}}$ is the normalized field amplitude of pump field $j, f_{R}$ is the fraction of the nonlinearity due to molecular vibrations given in Eq. (A17), $h_{R}$ is the normalized Raman response function, and $\omega_{S}$ and $\omega_{p_{j}}$ are the carrier frequencies of the Stokes and pump fields, respectively. The first term on the left-hand side describes the evolution of the Stokes field amplitude with distance $z$. The summation terms on the left hand side are responsible for the effect of dispersion on the Stokes field. The last term on the left-hand side describes the optical loss in the fiber for the Stokes field. The terms in the bracket on the right hand side describe the Kerrinduced contribution to self-phase modulation and crossphase modulation, respectively. The terms in the first integral on the right-hand side describe the molecular contribution to self-phase-modulation and cross-phase-modulation, respectively. The final summation terms are responsible for Raman amplification. Equation (1) is valid for Stokes pulse widths up to the limit of the slowly varying envelope approximation $(\sim 10 \mathrm{fs})$.

The first two integrals in Eq. (1) can further be simplified for longer pulse widths. For the Stokes and pump pulse widths on the order of $1 \mathrm{ps}, u_{S}$ and $u_{p_{j}}$ can be approximated to be constants compared with the time scale under which the Raman response function $h_{R}(t)$ is varying, such that Eq. (1) becomes

$$
\begin{aligned}
& \frac{\partial u_{S}}{\partial z}-i \sum_{m=1}^{\infty} \frac{i^{m} \beta_{m}}{m !} \frac{\partial^{m} u_{S}}{\partial t^{m}}+\frac{\alpha_{S}}{2} u_{S} \\
& =i \gamma_{S}\left(1-f_{R}\right) u_{S}\left[\left|u_{S}(t)\right|^{2}+2 \sum_{j=1}^{\infty}\left|u_{p_{j}}(t)\right|^{2}\right] \\
& \quad+i \gamma_{S} f_{R} u_{S}\left[\left|u_{S}(t)\right|^{2}+\sum_{j=1}^{\infty}\left|u_{p_{j}}(t)\right|^{2}\right] \int_{-\infty}^{\infty} h_{R}\left(t-t^{\prime}\right) d t^{\prime}
\end{aligned}
$$




$$
+i \gamma_{S} f_{R} u_{S}(t) \sum_{j=1}^{\infty}\left|u_{p_{j}}\right|^{2} \int_{-\infty}^{\infty} h_{R}\left(t-t^{\prime}\right) e^{-i\left(\omega_{p_{j}}-\omega_{S}\right)\left(t-t^{\prime}\right)} d t^{\prime} .
$$

Furthermore, since $\int_{-\infty}^{\infty} h_{R}\left(t-t^{\prime}\right) d t^{\prime}=1$ and $\tilde{h}_{R}\left(\omega_{p_{j}}\right)=\int_{-\infty}^{\infty} h_{R}(t$ $\left.-t^{\prime}\right) e^{-i\left(\omega_{p_{j}}-\omega_{S}\right)\left(t-t^{\prime}\right)} d t^{\prime}$ based on the definition of Fourier transformation

$$
u_{S}(z, t)=\frac{1}{2 \pi} \int_{-\infty}^{\infty} \tilde{u}_{S}\left(z, \omega-\omega_{S}\right) e^{-i\left(\omega-\omega_{S}\right) t} d \omega,
$$

Equation (2) becomes

$$
\begin{aligned}
& \frac{\partial u_{S}}{\partial z}-i \sum_{m=1}^{\infty} \frac{i^{m} \beta_{m}}{m !} \frac{\partial^{m} u_{S}}{\partial t^{m}}+\frac{\alpha_{S}}{2} u_{S} \\
& =i \gamma_{S} u_{S}\left[\left|u_{S}(t)\right|^{2}+\left(2-f_{R}\right) \sum_{j=1}^{\infty}\left|u_{p_{j}}(t)\right|^{2}\right] \\
& +i \gamma_{S} f_{R} u_{S}(t) \sum_{j=1}^{\infty}\left|u_{p_{j}}\right|^{2} \tilde{h}_{R}\left(\omega_{p_{j}}\right) .
\end{aligned}
$$

The real part of $\widetilde{h}_{R}\left(\omega_{p_{j}}\right)$ is responsible for the Raman-induced index change whereas the imaginary part of $\tilde{h}_{R}\left(\omega_{p_{j}}\right)$ corresponds to the Raman amplification. Rewriting Eq. (4) in terms of the real and imaginary parts of $\tilde{h}_{R}\left(\omega_{p_{j}}\right)$ with $\tilde{h}_{R}\left(\omega_{p_{j}}\right)=\operatorname{Re}\left[\tilde{h}_{R}\left(\omega_{p_{j}}\right)\right]+i \operatorname{Im}\left[\tilde{h}_{R}\left(\omega_{p_{j}}\right)\right]$ and $\tilde{h}_{R}\left(\omega_{p_{j}}\right)$ $=-\tilde{h}_{R}\left(-\omega_{p_{j}}\right)$, which is a symmetric function, and assuming that the pump fields are much more energetic than the Stokes field so that $\left|u_{S}\right| \ll\left|u_{p_{j}}\right|$, we find

$$
\begin{aligned}
& \frac{\partial u_{S}}{\partial z}-i \sum_{m=1}^{\infty} \frac{i^{m} \beta_{m}}{m !} \frac{\partial^{m} u_{S}}{\partial t^{m}}+\frac{\alpha_{S}}{2} u_{S} \\
& =i \gamma_{S} u_{S} \sum_{j=1}^{\infty}\left[2+f_{R} \operatorname{Re}\left[\tilde{h}_{R}\left(\omega_{p_{j}}\right)\right]-f_{R}\right]\left|u_{p_{j}}(t)\right|^{2} \\
& +\gamma_{S} f_{R} u_{S} \sum_{j=1}^{\infty}\left|u_{p_{j}}(t)\right|^{2} \operatorname{Im}\left[\tilde{h}_{R}\left(-\omega_{p_{j}}\right)\right] .
\end{aligned}
$$

Moving into the frequency domain based on the definition of Fourier transformation in Eq. (3) and assuming that the pump fields are undepleted such that $u_{p_{j}}$ are constants independent from $t$ and $z$, Eq. (5) is found to be a simple differential equation with respect to $z$,

$$
\begin{aligned}
& \frac{1}{2 \pi} \int_{-\infty}^{\infty} d \omega\left\{\frac{\partial}{\partial z}-i \sum_{m=1}^{\infty} \frac{\beta_{m}}{m !}\left(\omega-\omega_{S}\right)^{m}\right. \\
& \quad+\frac{\alpha_{S}}{2}-i \gamma_{S} \sum_{j=1}^{\infty}\left[2+f_{R} \operatorname{Re}\left[\tilde{h}_{R}\left(\omega_{p_{j}}\right)\right]-f_{R}\right]\left|u_{p_{j}}\right|^{2} \\
& \left.\quad-\gamma_{S} f_{R} \sum_{j=1}^{\infty}\left|u_{p_{j}}\right|^{2} \operatorname{Im}\left[\tilde{h}_{R}\left(-\omega_{p_{j}}\right)\right]\right\} \tilde{u}_{S}\left(\omega-\omega_{S}\right) e^{-i\left(\omega-\omega_{S}\right) t}=0,
\end{aligned}
$$

which indicates that the integrand is equal to zero. Thus, the solution of the equation is found to be

$$
\begin{aligned}
\tilde{u}_{S}\left(z, \omega-\omega_{S}\right)= & \tilde{u}_{S}\left(0, \omega-\omega_{S}\right) \exp \left\{i \sum_{m=1}^{\infty} \frac{\beta_{m}}{m !}\left(\omega-\omega_{S}\right)^{m} z-\frac{\alpha_{S}}{2} z\right. \\
& +i \gamma_{S} \sum_{j=1}^{\infty}\left[2+f_{R} \operatorname{Re}\left[\tilde{h}_{R}\left(\omega_{p_{j}}\right)\right]-f_{R}\right]\left|u_{p_{j}}\right|^{2} z \\
& \left.+\gamma_{S} f_{R} \sum_{j=1}^{\infty}\left|u_{p_{j}}\right|^{2} \operatorname{Im}\left[\tilde{h}_{R}\left(-\omega_{p_{j}}\right)\right] z\right\} .
\end{aligned}
$$

Substituting this into Eq. (3) gives the function that describes the generalized amplified dispersive Fourier transformation,

$$
\begin{aligned}
u_{S}(z, t)= & \frac{1}{2 \pi} \int_{-\infty}^{\infty} d \omega \tilde{u}_{S}\left(0, \omega-\omega_{S}\right) \exp \left\{i \sum_{m=1}^{\infty} \frac{\beta_{m}}{m !}\left(\omega-\omega_{S}\right)^{m} z\right. \\
& -\frac{\alpha_{S}}{2} z+i \gamma_{S} \sum_{j=1}^{\infty}\left[2+f_{R} \operatorname{Re}\left[\tilde{h}_{R}\left(\omega_{p_{j}}\right)\right]-f_{R}\right]\left|u_{p_{j}}\right|^{2} z \\
& \left.+\gamma_{S} f_{R} \sum_{j=1}^{\infty}\left|u_{p_{j}}\right|^{2} \operatorname{Im}\left[\tilde{h}_{R}\left(-\omega_{p_{j}}\right)\right] z-i\left(\omega-\omega_{S}\right) t\right\},
\end{aligned}
$$

which indicates how the input Stokes spectrum $\tilde{u}_{S}(0, \omega$ $\left.-\omega_{S}\right)$ transforms into the temporal output Stokes field $u_{S}(z, t)$.

In general, the Raman gain spectrum of the silica fiber is given by $\operatorname{Im}\left[\tilde{h}_{R}\left(\omega_{p_{j}}\right)\right][14]$. Assuming that the total Raman gain spectrum is designed to be flat over a bandwidth of $\Delta \omega$ centered at $\Omega_{S}$ by the use of multiple pump fields with different powers at different center wavelengths $[1,4,5,15,16]$ so that $\sum_{j=1}^{\infty} \mid u_{p_{j}}{ }^{2} \operatorname{Im}\left[\tilde{h}_{R}\left(-\omega_{p_{j}}\right)\right]=$ constant and $\operatorname{Re}\left[\tilde{h}_{R}\left(\omega_{p_{j}}\right)\right]$ $\simeq 0$ for $\Omega_{S}-\Delta \omega / 2 \leq \omega \leq \Omega_{S}+\Delta \omega / 2$, Eq. (8) can be approximated to be

$$
\begin{aligned}
u_{S}(z, t) \simeq & \frac{1}{2 \pi} \exp \left[-\frac{\alpha_{S}}{2} z+i \gamma_{S} \sum_{j=1}^{\infty}\left(2-f_{R}\right) \mid u_{p_{j}}{ }^{2} z+\frac{g_{S}}{2} z\right] \\
& \times \int_{\Omega_{S}-\Delta \omega / 2}^{\Omega_{S}+\Delta \omega / 2} \tilde{u}_{S}\left(0, \omega-\omega_{S}\right) \\
& \times \exp \left[i \sum_{m=1}^{\infty} \frac{\beta_{m}}{m !}\left(\omega-\omega_{S}\right)^{m} z-i\left(\omega-\omega_{S}\right) t\right] d \omega,
\end{aligned}
$$

where we have defined $g_{S}$ to be

$$
g_{S}=2 \gamma_{S} f_{R} \sum_{j=1}^{\infty}\left|u_{p_{j}}\right|^{2} \operatorname{Im}\left[\tilde{h}_{R}\left(-\omega_{p_{j}}\right)\right] .
$$

Rewriting Eq. (9) in terms of the time in a frame of reference moving with the Stokes pulse at the group velocity $1 / \beta_{1}$, we find 


$$
\begin{aligned}
u_{S}(z, t) \simeq & \frac{1}{2 \pi} \exp \left[-\frac{\alpha_{S}}{2} z+i \gamma_{S} \sum_{j=1}^{\infty}\left(2-f_{R}\right)\left|u_{p_{j}}\right|^{2} z+\frac{g_{S}}{2} z\right] \\
& \times \int_{\Omega_{S}-\Delta \omega / 2}^{\Omega_{S}+\Delta \omega / 2} \widetilde{u}_{S}\left(0, \omega-\omega_{S}\right) \\
& \times \exp \left[i \sum_{m=2}^{\infty} \frac{\beta_{m}}{m !}\left(\omega-\omega_{S}\right)^{m} z-i\left(\omega-\omega_{S}\right) T\right] d \omega,
\end{aligned}
$$

where

$$
T=t-\beta_{1} z
$$

To examine the evolution of the input spectrum $\widetilde{u}_{S}(0, \omega$ $-\omega_{S}$ ) for large dispersion, we consider the following two limiting cases and then generalize ADFT.

\section{A. Amplified dispersive Fourier transformation with up to the second-order GVD coefficients}

Taking into account the GVD coefficients only up to the second-order, the integral in Eq. (11) becomes

$$
\begin{aligned}
\Psi(z, T)= & \int_{\Omega_{S^{-}-\Delta \omega / 2}}^{\Omega_{S}+\Delta \omega / 2} \widetilde{u}_{S}\left(0, \omega-\omega_{S}\right) \\
& \times \exp \left[i \frac{\beta_{2}}{2}\left(\omega-\omega_{S}\right)^{2} z-i\left(\omega-\omega_{S}\right) T\right] d \omega,
\end{aligned}
$$

which can be written as

$$
\begin{aligned}
\Psi(z, T)= & \exp \left(-i \frac{T^{2}}{2 \beta_{2} z}\right) \int_{\Omega_{S^{-}-\omega / 2}}^{\Omega_{S^{+} \Delta \omega / 2}} \tilde{u}_{S}\left(0, \omega-\omega_{S}\right) \\
& \times \exp \left[i \frac{\beta_{2} z}{2}\left(\omega-\omega_{S}-\frac{T}{\beta_{2} z}\right)^{2}\right] d \omega,
\end{aligned}
$$

For large $\beta_{2} z$ (large GVD), the integrand oscillates rapidly in the frequency domain except for the frequency $\omega=\Omega_{S}$, where

$$
\Omega_{S}=\omega_{S}+\frac{T}{\beta_{2} z},
$$

which indicates that $\Omega_{S}$ and $T$ have the linear relation. This equation can be rewritten as

$$
T=\beta_{2} z\left(\Omega_{S}-\omega_{S}\right) .
$$

To solve the integral in Eq. (14), we apply the stationaryphase approximation to it. It is an approach for solving integrals analytically by evaluating the integrands in regions where they contribute the most. In this case, the stationaryphase approximation assumes that the input spectrum $\widetilde{u}_{S}\left(0, \omega-\omega_{S}\right)$ is a slowly varying function while the exponential in the integral is a rapidly varying function. As a result, $\widetilde{u}_{S}\left(0, \omega-\omega_{S}\right)$ is approximated to be constant and can be factored out of the integral by evaluating it at $\omega=\Omega_{S}$. Then, the equation becomes
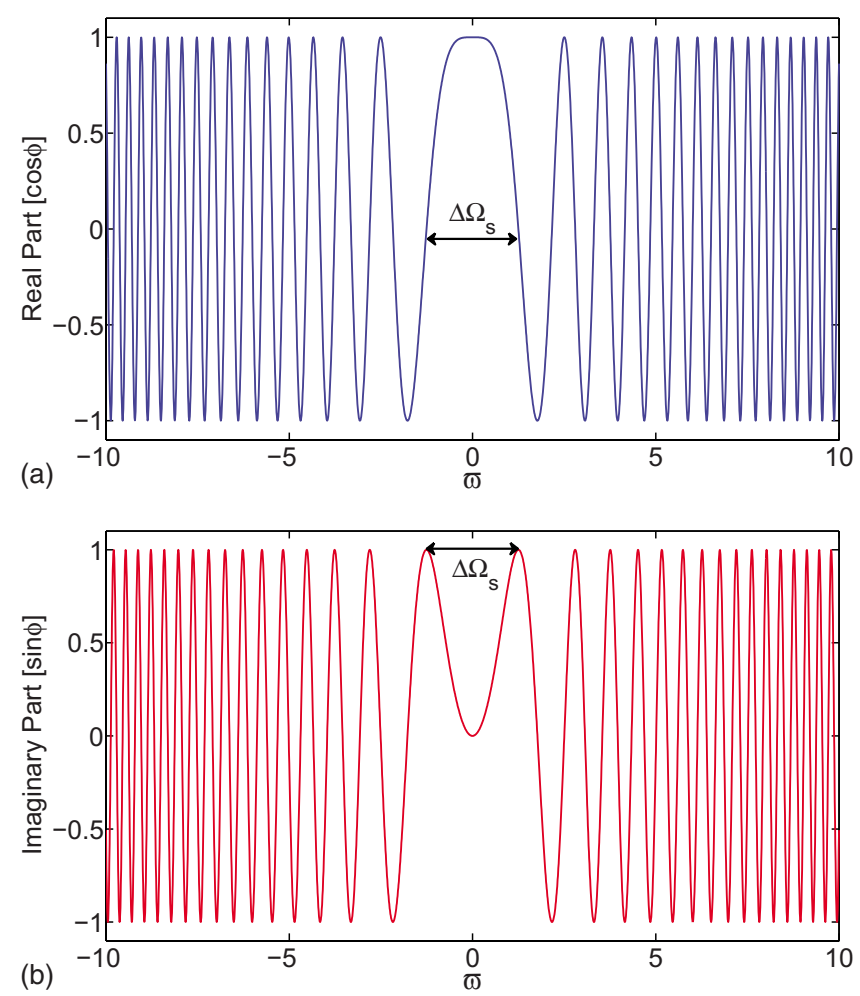

FIG. 2. (Color online) The real (top) and imaginary (bottom) parts of the exponential in the integral in Eq. (17). $\phi$ is defined to be $\phi=\beta_{2} z\left(\omega-\Omega_{S}\right)^{2} / 2=a \varpi^{2}$, where $a=\beta_{2} z \Omega_{S}^{2} / 2$ and $\varpi=\omega / \Omega_{S}-1$, which is the normalized frequency. $a=1$ is used for the figure. Both $\cos \phi$ and $\sin \phi$ oscillate rapidly as $\varpi$ varies from the origin. Only the frequencies in the central region of the two plots contribute to the temporal waveform at a particular time instant. Contributions from all the other frequencies oscillate rapidly between positive and negative values and cancel out when integrated over all frequencies.

$$
\begin{aligned}
\Psi(z, T) \simeq & \tilde{u}_{S}\left(0, \Omega_{S}-\omega_{S}\right) \exp \left(-i \frac{T^{2}}{2 \beta_{2} z}\right) \\
& \times \int_{\Omega_{S^{-}-\Delta \omega / 2}}^{\Omega_{S^{+} \Delta \omega / 2}} \exp \left\{i \frac{\beta_{2} z}{2}\left[\omega-\Omega_{S}(T)\right]^{2}\right\} d \omega .
\end{aligned}
$$

The assumption for the stationary-phase approximation is analogous to the requirement for the far-field approximation in paraxial diffraction.

The real and imaginary parts of the exponential in the integral in Eq. (17) are shown in Fig. 2. In the figure, $\phi$ is defined to be $\phi=\beta_{2} z\left(\omega-\Omega_{S}\right)^{2} / 2=a \varpi^{2}$, where $a=\beta_{2} z \Omega_{S}^{2} / 2$ and $\varpi=\omega / \Omega_{S}-1$, which is a normalized frequency parameter. Both the real $(\cos \phi)$ and imaginary $(\sin \phi)$ parts of the exponential oscillate rapidly as $\varpi$ varies from the origin. Here it is important to note that since rapid oscillations between positive and negative values take place as the frequency $\omega$ varies relative to $\Omega_{S}$, only the central part of $\cos \phi$ and $\sin \phi$ survives in the integral. The width of this central part in Fig. 2 can be obtained by defining the width $\Delta \Omega_{S}$ to be the range between the first zeros of the real part of the exponential $(\cos \phi)$ or the two central peaks of the imaginary part $(\sin \phi)$, which occur at $\phi=\pi / 2$, 


$$
\frac{\beta_{2} z}{2}\left(\Omega_{S} \pm \frac{\Delta \Omega_{S}}{2}-\omega_{S}-\frac{T}{\beta_{2} z}\right)^{2}=\frac{\pi}{2}
$$

from which $\Delta \Omega_{S}$ is found to be

$$
\Delta \Omega_{S}=2 \sqrt{\frac{\pi}{\beta_{2} z}} .
$$

For large $\beta_{2} z$ (large GVD), the spectral width decreases, as expected. Here it is important to realize that the spectral width $\Delta \Omega_{S}$ is the width of the slowly varying central peak in the real part $(\cos \phi)$ of the exponential around the frequency $\Omega_{S}$ in which the integrand survives, as shown in Fig. 2. For frequencies outside of the width, the exponential in the integral oscillates rapidly between positive and negative values and its integral vanishes. For one-to-one frequency-to-time mapping to occur - that is, the ideal situation - only one frequency would contribute to the temporal waveform at any one time instant. In other words, the central peak in Fig. 2 would have an infinitesimal width. Therefore, the width of this peak is a measure of the ambiguity in the frequency-totime mapping (i.e., dispersive Fourier transform) process, which may be used as a measure of the resolution of the dispersive Fourier transform spectrometer. We caution that this is not a precise or hard limit on the closest frequencies that can be resolved by this technique. Smaller differences can be distinguished, but the temporal waveform will not be an exact replica of the optical spectrum, with the relative deviation increasing for more closely spaced frequencies.

Using the spectral width $\Delta \Omega_{S}$, Eq. (17) is then calculated to be

$$
\Psi(z, T) \simeq \widetilde{u}_{S}\left(0, \Omega_{S}-\omega_{S}\right) \exp \left(-i \frac{T^{2}}{2 \beta_{2} z}\right)(1+i) \Delta \Omega_{S} .
$$

Here it is important to understand that $\Omega_{S}$, as shown in Eq. (15), is a function of $T$, satisfying $\Omega_{S}=\omega_{S}+T /\left(\beta_{2} z\right)$, so that the integral is nonvanishing only when $\omega$ is approximately equal to $\Omega_{S}$. Since $\Omega_{S}$ varies with $T$, the temporal equivalent of $\Delta \Omega_{S}$ is given by

$$
\Delta T=\frac{d T}{d \Omega_{S}} \Delta \Omega_{S}=2 \sqrt{\pi \beta_{2} z}
$$

where we have used Eq. (19). This equation indicates that as the total GVD increases, the requirement on the bandwidth (i.e., the temporal resolution) of the optical intensity detection system (which consists of a photodetector and digitizer or oscilloscope) becomes relaxed.

Substituting Eqs. (19) and (20) back into Eq. (11), we find the absolute value of the optical field (i.e., the square root of the measured optical intensity) to be proportional to

$$
\left|u_{S}(z, T)\right|=\sqrt{\frac{2}{\pi \beta_{2} z}} e^{1 / 2\left(g_{S}-\alpha_{S}\right) z}\left|\widetilde{u}_{S}\left(0, \Omega_{S}-\omega_{S}\right)\right|,
$$

which indicates the mapping of the spectrum of the input Stokes pulse into the time-domain waveform.

\section{B. Amplified dispersive Fourier transformation with up to the third-order GVD coefficients}

Taking into account the GVD coefficients up to the thirdorder, the integral in Eq. (11) becomes

$$
\begin{aligned}
\Psi(z, T)= & \int_{\Omega_{S^{-}-\Delta \omega / 2}}^{\Omega_{S^{+}+\Delta \omega / 2}} \tilde{u}_{S}\left(0, \omega-\omega_{S}\right) \exp \left[i \frac{\beta_{2}}{2}\left(\omega-\omega_{S}\right)^{2} z\right. \\
& \left.+i \frac{\beta_{3}}{6}\left(\omega-\omega_{S}\right)^{3} z-i\left(\omega-\omega_{S}\right) T\right] d \omega .
\end{aligned}
$$

The exponent in the integral can be written as

$$
\begin{aligned}
\frac{i}{2} \beta_{2} & \left(\omega-\omega_{S}\right)^{2} z+\frac{i}{6} \beta_{3}\left(\omega-\omega_{S}\right)^{3} z-i\left(\omega-\omega_{S}\right) T \\
= & \frac{i}{6} \beta_{3} z\left[\omega-\omega_{S}+\frac{\beta_{2}}{\beta_{3}}+2 \sqrt{\left(\frac{\beta_{2}}{\beta_{3}}\right)^{2}+\frac{2 T}{\beta_{3} z}}\right]\left[\omega-\omega_{S}+\frac{\beta_{2}}{\beta_{3}}\right. \\
& \left.-\sqrt{\left(\frac{\beta_{2}}{\beta_{3}}\right)^{2}+\frac{2 T}{\beta_{3} z}}\right]^{2}-\frac{i}{6} \beta_{3} z\left[\frac{\beta_{2}}{\beta_{3}}+2 \sqrt{\left(\frac{\beta_{2}}{\beta_{3}}\right)^{2}+\frac{2 T}{\beta_{3} z}}\right] \\
& \times\left[\frac{\beta_{2}}{\beta_{3}}-\sqrt{\left(\frac{\beta_{2}}{\beta_{3}}\right)^{2}+\frac{2 T}{\beta_{3} z}}\right]^{2} .
\end{aligned}
$$

For large $\beta_{3} z$ (large GVD), the integrand oscillates rapidly in the frequency domain except for the frequency $\omega=\Omega_{S}$, where

$$
\Omega_{S}=\omega_{S}-\frac{\beta_{2}}{\beta_{3}}+\sqrt{\left(\frac{\beta_{2}}{\beta_{3}}\right)^{2}+\frac{2 T}{\beta_{3} z}},
$$

which indicates that $\Omega_{S}$ and $T$ have a nonlinear relation. As $T \ll \beta_{2}^{2} z /\left(2 \beta_{3}\right)$, the equation can approximated to be

$$
\Omega_{S} \simeq \omega_{S}+\frac{T}{\beta_{2} z}
$$

which is equivalent to Eq. (15), as expected. Equation (25) can be rewritten as

$$
T=\beta_{2} z\left(\Omega_{S}-\omega_{S}\right)+\frac{1}{2} \beta_{3} z\left(\Omega_{S}-\omega_{S}\right)^{2} .
$$

As demonstrated above, for large $\beta_{3} z$ (large GVD), applying the stationary-phase approximation to the integral gives

$$
\begin{aligned}
\Psi(z, T) \simeq & \exp \left\{-\frac{i}{6} \beta_{3} z\left[\frac{\beta_{2}}{\beta_{3}}+2 \sqrt{\left(\frac{\beta_{2}}{\beta_{3}}\right)^{2}+\frac{2 T}{\beta_{3} z}}\right]\left[\frac{\beta_{2}}{\beta_{3}}\right.\right. \\
& \left.\left.-\sqrt{\left(\frac{\beta_{2}}{\beta_{3}}\right)^{2}+\frac{2 T}{\beta_{3} z}}\right]^{2}\right\} \widetilde{u}_{S}\left(0, \Omega_{S}-\omega_{S}\right)(1+i) \Delta \Omega_{S},
\end{aligned}
$$

where $\Delta \Omega_{S}$ is given by setting the phase in the integral 


$$
\begin{aligned}
\frac{\beta_{3} z}{6}[ & \left.\Omega_{S} \pm \frac{\Delta \Omega_{S}}{2}-\omega_{S}+\frac{\beta_{2}}{\beta_{3}}+2 \sqrt{\left(\frac{\beta_{2}}{\beta_{3}}\right)^{2}+\frac{2 T}{\beta_{3} z}}\right] \\
& \times\left[\Omega_{S} \pm \frac{\Delta \Omega_{S}}{2}-\omega_{S}+\frac{\beta_{2}}{\beta_{3}}-\sqrt{\left(\frac{\beta_{2}}{\beta_{3}}\right)^{2}+\frac{2 T}{\beta_{3} z}}\right]^{2}=\frac{\pi}{2},
\end{aligned}
$$

from which $\Delta \Omega_{S}$ is found to be

$$
\Delta \Omega_{S} \simeq 2 \sqrt{\frac{\pi}{\beta_{2} z+\beta_{3} z\left(\Omega_{S}-\omega_{S}\right)}},
$$

where we have assumed $\Delta \Omega_{S} \ll \Omega_{S}-\omega_{S}$. For small bandwidths, this reduces to Eq. (19), as expected. The temporal equivalence of the spectral resolution $\Delta \Omega_{S}$ in ADFT is also found to be

$$
\Delta T=\frac{d T}{d \Omega_{S}} \Delta \Omega_{S}=2 \sqrt{\pi\left[\beta_{2} z+\beta_{3} z\left(\Omega_{S}-\omega_{S}\right)\right]} .
$$

Similar to the calculations in Sec. II A, substituting Eqs. (28) and (30) back into Eq. (11) and taking its absolute value, we find

$$
\begin{aligned}
\left|u_{S}(z, T)\right|= & \sqrt{\frac{2}{\pi\left[\beta_{2} z+\beta_{3} z\left(\Omega_{S}-\omega_{S}\right)\right]}} \\
& \times e^{1 / 2\left(g_{S^{-}} \alpha_{S} z\right.}\left|\widetilde{u}_{S}\left(0, \Omega_{S}-\omega_{S}\right)\right|,
\end{aligned}
$$

which indicates the mapping of the spectrum of the input Stokes pulse into the time-domain waveform including the effect of the third-order GVD coefficient.

\section{Generalized amplified dispersive Fourier transformation with all the higher-order GVD coefficients}

As higher-order GVD coefficients are included in Eq. (11), it is no longer straightforward to derive the stationary phase and express $\Omega_{S}$ in terms of $T$ and the higher-order GVD coefficients analytically like in Eqs. (15) and (25). It is rather simple to express $T$ in terms of $\Omega_{S}$. Following Eqs. (16) and (27), we find the generalized frequency-time relation,

$$
T\left(\Omega_{S}\right)=\sum_{m=1}^{\infty} \frac{\beta_{m+1}}{m !}\left(\Omega_{S}-\omega_{S}\right)^{m} z .
$$

Similar to the calculations in Secs. II A and II B, following Eqs. (18) and (29), the generalized spectral width $\Delta \Omega_{S}$ can be found by mathematical induction,

$$
\Delta \Omega_{S} \simeq 2 \sqrt{\frac{\pi}{\sum_{m=0}^{\infty} \frac{\beta_{m+2}}{m !} z\left(\Omega_{S}-\omega_{S}\right)^{m}}},
$$

where we have assumed $\Delta \Omega_{S} \ll \Omega_{S}-\omega_{S}$ to derive Eq. (34). It is important to realize that this spectral width is equivalent to the spectral resolution of ADFT. As the amount of dispersion increases, the spectral resolution (as defined by the stationary-phase approximation) improves, as expected.
Also, Eqs. (21) and (31) can easily be extended to the case with all GVD coefficients. From Eqs. (33) and (34), we find the general temporal equivalent of the spectral resolution to be

$$
\Delta T=\frac{d T}{d \Omega_{S}} \Delta \Omega_{S}=2 \sqrt{\pi \sum_{m=0}^{\infty} \frac{\beta_{m+2}}{m !} z\left(\Omega_{S}-\omega_{S}\right)^{m}},
$$

which can be reduced to Eq. (21) when $\Omega_{S}-\omega_{S}$ is small, as expected.

Finally, the generalized ADFT including all the GVD coefficients and the generalized spectral width given by Eq. (34) is found to be

$$
\begin{aligned}
\left|u_{S}(z, T)\right|= & \sqrt{\frac{2}{\pi\left[\sum_{m=0}^{\infty} \frac{\beta_{m+2}}{m !} z\left(\Omega_{S}-\omega_{S}\right)^{m}\right]}} \\
& \times e^{1 / 2\left(g_{S}-\alpha_{S}\right)}\left|\tilde{u}_{S}\left(0, \Omega_{S}-\omega_{S}\right)\right|,
\end{aligned}
$$

which indicates the mapping of the spectrum of the input Stokes pulse $\left|\widetilde{u}_{S}\left(0, \Omega_{S}-\omega_{S}\right)\right|$ into the time-domain waveform $\left|u_{S}(z, T)\right|$ with all the GVD coefficients included, as expected.

\section{ANALYSIS}

In Sec. II, we have derived the general equation for ADFT from the generalized nonlinear Schrödinger equation with all GVD coefficients in the presence of multiple pump fields. We have also derived a measure for the spectral resolution in ADFT as well as its temporal equivalent. In this section, based on the derived equations, we examine how the spectrum of an optical pulse evolves into a time-domain waveform, the effect of linear and nonlinear GVD on ADFT, and the requirement for dispersion to perform the stationaryphase approximation that enables ADFT in order to analyze the accuracy of ADFT. The effect of noise associated with optical amplification on ADFT, which is equally important, will be discussed in a separate paper. As the dispersion parameters are more commonly used to describe dispersive properties of optical fibers, we use the parameters instead of the GVD coefficients throughout this section. The relations between the dispersion parameters and the GVD coefficients are described in Appendix B.

\section{A. Evolution of the spectrum of an optical pulse into a temporal waveform}

Figures 3 and 4 show the evolution of the spectrum of an optical pulse into a temporal waveform in ADFT whose general equation is given by Eq. (36). Both figures show an input spectrum and the ADFT waveform of the input spectrum that is mapped into the time domain for various dispersion values. The input spectrum in Fig. 3 is the sum of two Gaussian lines, which can only be resolved when sufficient dispersion is used in ADFT. Figure 4 shows the ADFT waveform of two absorption lines on top of a frequency comb of delta functions with equal amplitude and comb spacing. In both examples, which are often used in practical applica- 

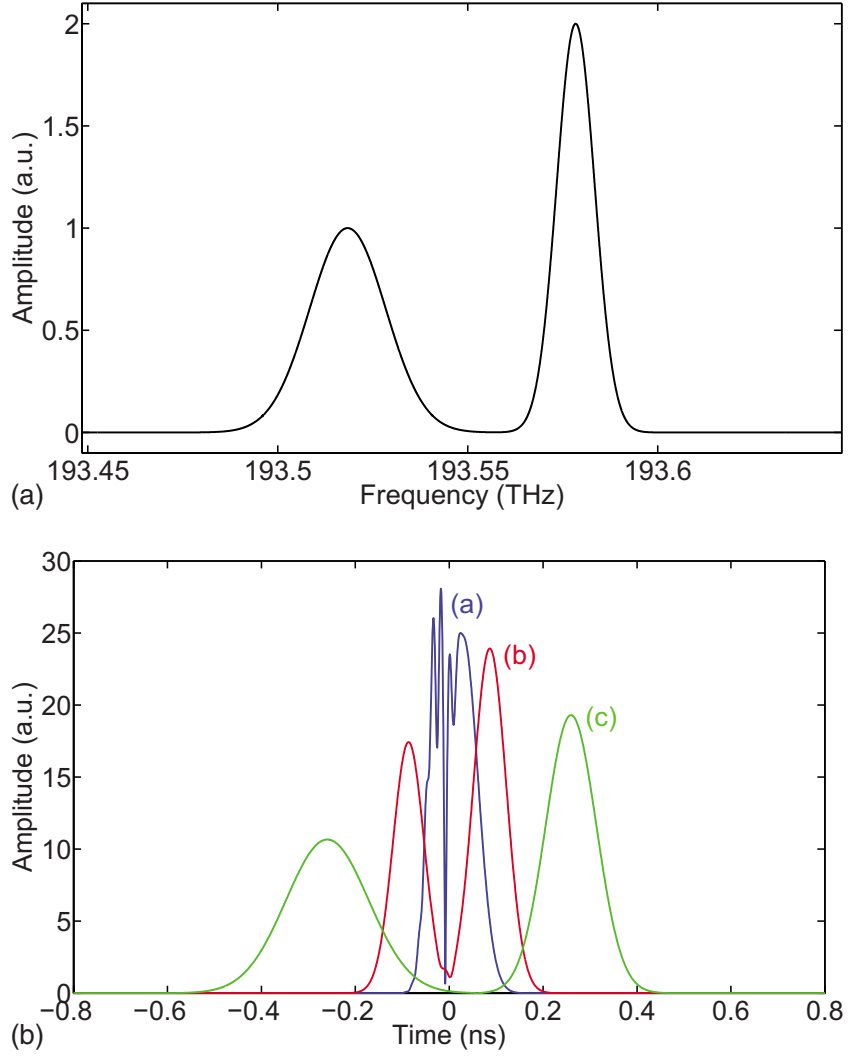

FIG. 3. (Color online) Top: An input spectrum which is the sum of two Gaussian lines. Bottom: The ADFT waveform of the input spectrum that is mapped into the time domain for various amounts of GVD: (a) $1 \mathrm{~km}$, (b) $3 \mathrm{~km}$, and (c) $9 \mathrm{~km}$. The values used for the dispersion parameter and dispersion slope are $-120(\mathrm{ps} / \mathrm{nm}) / \mathrm{km}$ and $0.3\left(\mathrm{ps} / \mathrm{nm}^{2}\right) / \mathrm{km}$, respectively. The center wavelength is 1550 $\mathrm{nm}$. As the amount of GVD increases, the input pulse is transformed into a temporal waveform which resembles the input spectrum. It is important to note that the two Gaussian lines can only be resolved when sufficient GVD is used for ADFT.

tions, the ADFT waveform of an input spectrum in the time domain matches the input spectrum in the frequency domain.

\section{B. Effect of higher-order GVD coefficients}

As shown in Eq. (16), when the higher-order GVD coefficients are not considered in the case of small bandwidths, the frequency-time relation is linear. However, for large bandwidths, their effect on the frequency-time relation needs to be taken into account, and hence, the frequency-to-time transformation must be recalibrated. Figure 5 shows the effect of $\beta_{3}$ and the bandwidth $\Omega_{S}-\omega_{S}$ on the frequency-time relation given in Eq. (33). In the figure, the frequency-time relation is plotted as a function of the zeroth-order and firstorder dispersion parameters instead of the GVD coefficients.

\section{Dependence on the amount of GVD}

As discussed in Sec. II, ADFT is based on the stationaryphase approximation enabled by large GVD. If the amount of GVD is insufficient, the stationary-phase approximation does not hold. As a result, fine spectral features are distorted or
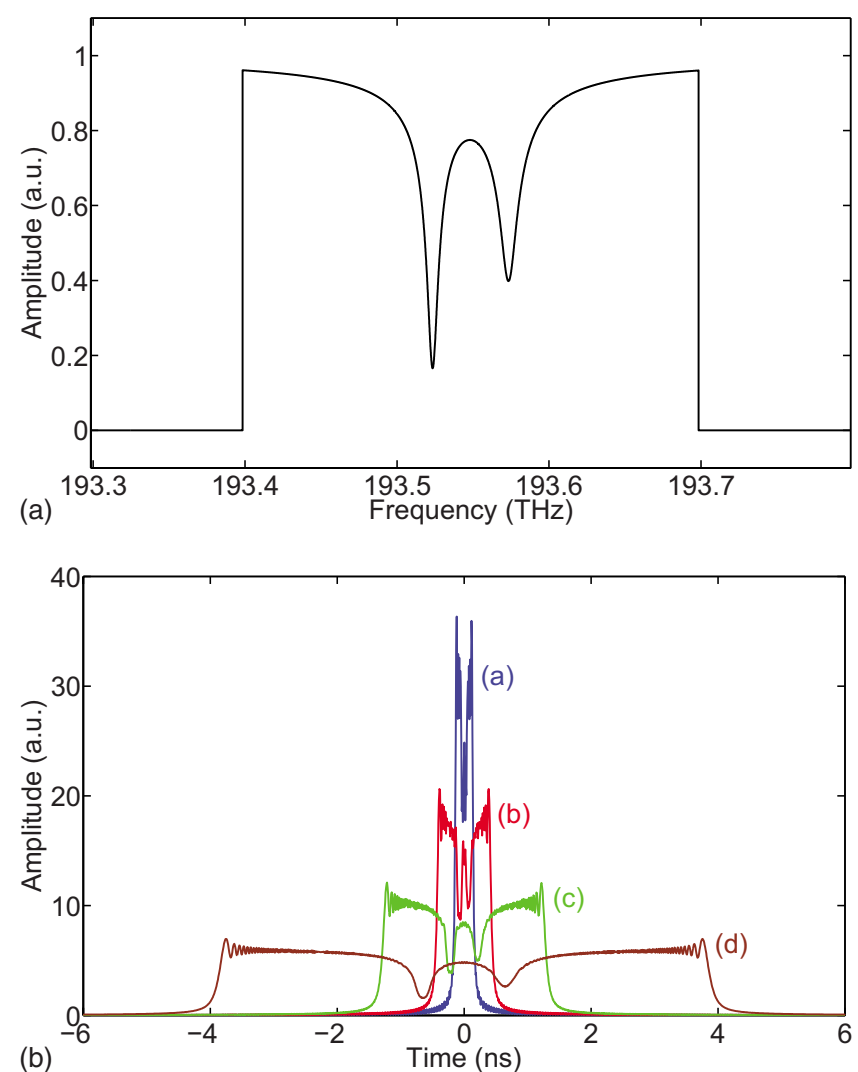

FIG. 4. (Color online) Top: An input spectrum which is the sum of two Lorentzian-shaped absorption lines with a continuum background. Bottom: The ADFT waveform of the input spectrum that is mapped into the time domain for various dispersion lengths: (a) 1, (b) 3, (c) 9, and (d) $27 \mathrm{~km}$. The values used for the dispersion parameter and dispersion slope are $-120(\mathrm{ps} / \mathrm{nm}) / \mathrm{km}$ and $0.3\left(\mathrm{ps} / \mathrm{nm}^{2}\right) / \mathrm{km}$, respectively. The center wavelength is $1550 \mathrm{~nm}$. As the amount of GVD increases, the input pulse is transformed into a temporal waveform which resembles the input spectrum. It is important to note that the two absorption lines can only be resolved when sufficient GVD is used for ADFT. The ripples on top of the rectangular spectrum shape are less evident for larger GVD, indicating that the transformation is near completion. The ripples at the edges are due to the sharp edges of the input spectrum.

lost when the intensity of the dispersed optical field is captured. Based on the stationary-phase approximation, the spectral resolution or ambiguity defined as the width of the central frequency peak in Fig. 2 is given by Eq. (34). This measure of the spectral resolution improves with increase in the total GVD. With more GVD, finer spectral resolution can be achieved. This is observed in Fig. 3 in which the two Gaussian spectral lines can only be resolved with sufficiently large GVD. Figure 6 shows the spectral resolution given in Eq. (34) as the amount of dispersion varies. The spectral resolution improves with larger GVD, as expected. For example, for typical dispersion compensation fibers which are commercially available, the dispersion parameter and dispersion slope are $-120(\mathrm{ps} / \mathrm{nm}) / \mathrm{km}$ and $0.3\left(\mathrm{ps} / \mathrm{nm}^{2}\right) / \mathrm{km}$, yielding a spectral resolution of about $4.5 \mathrm{GHz}$ for a dispersion length of $100 \mathrm{~km}$. Furthermore, the effect of $\beta_{3}$ and the bandwidth on the spectral resolution given in Eq. (34) is 


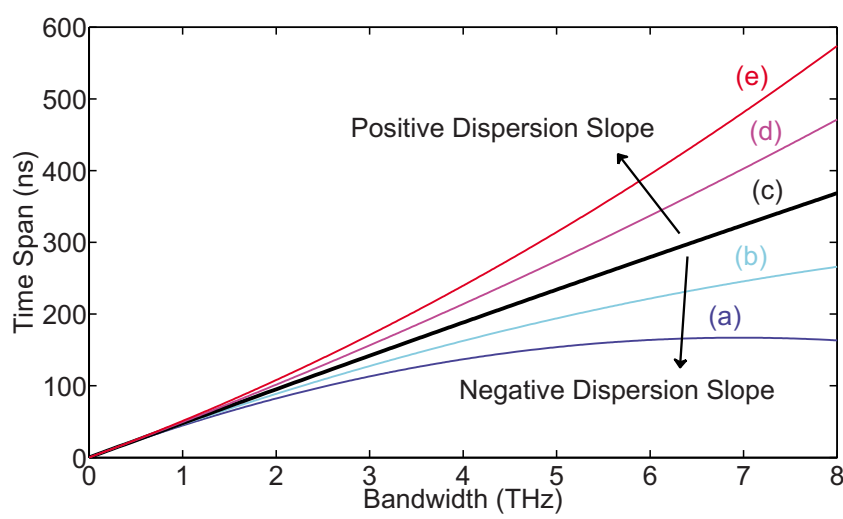

FIG. 5. (Color online) The effect of $\beta_{3}$ and the bandwidth $\Omega_{S}$ $-\omega_{S}$ on the frequency-time relation given in Eq. (33) for various values of $d D /\left.d \lambda\right|_{\lambda_{S}}$ (the relation between the GVD coefficients and dispersion parameters is given in Appendix B): (a) $-2\left(\mathrm{ps} / \mathrm{nm}^{2}\right) / \mathrm{km}$, (b) $-1\left(\mathrm{ps} / \mathrm{nm}^{2}\right) / \mathrm{km}$, (c) $0\left(\mathrm{ps} / \mathrm{nm}^{2}\right) / \mathrm{km}$, (d) $1\left(\mathrm{ps} / \mathrm{nm}^{2}\right) / \mathrm{km}$, and (e) $2\left(\mathrm{ps} / \mathrm{nm}^{2}\right) / \mathrm{km}$. The dispersion length, the center wavelength, and the dispersion parameter $D\left(\lambda_{S}\right)$ used for the figure are $z=50 \mathrm{~km}, 1550 \mathrm{~nm}$, and $-120(\mathrm{ps} / \mathrm{nm}) / \mathrm{km}$, respectively. The higher-order dispersion parameters are not considered here. It is important to note that in absence of the dispersion slope (or when the dispersion parameter is constant), the frequency-time relation is completely linear regardless of the bandwidth. Otherwise, the frequency-time relation is nonlinear and hence needs to be recalibrated. The effect of $\beta_{3}$ becomes evident with larger bandwidth.

shown in Fig. 7. The spectral resolution improves with the positive dispersion slope.

\section{Requirement for the bandwidth of the electronic digitizer}

In practice, we need to take into account the temporal resolution of the optical signal detection system because it can limit the spectral resolution. The optical intensity detec-

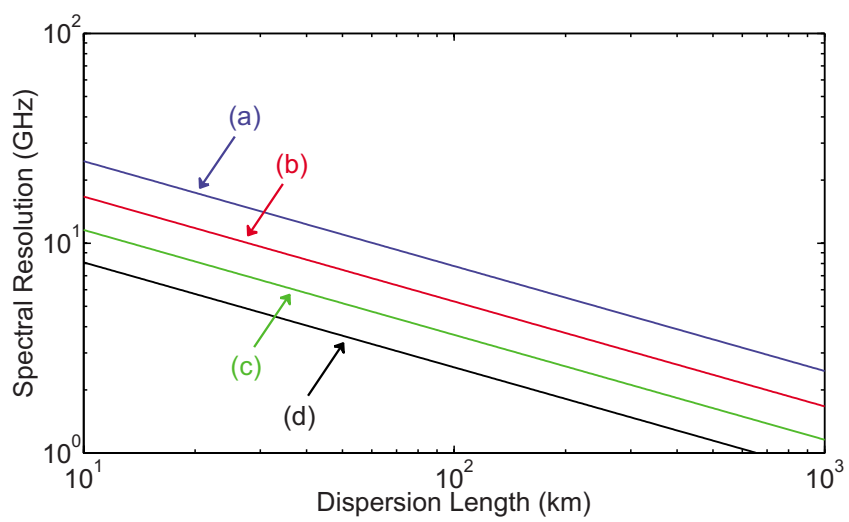

FIG. 6. (Color online) The spectral resolution given in Eq. (34) as the dispersion length varies for various dispersion parameter values: (a) $-50(\mathrm{ps} / \mathrm{nm}) / \mathrm{km}, \quad$ (b) $-100(\mathrm{ps} / \mathrm{nm}) / \mathrm{km}, \quad$ (c) $-200(\mathrm{ps} / \mathrm{nm}) / \mathrm{km}$, and (d) $-400(\mathrm{ps} / \mathrm{nm}) / \mathrm{km}$. The dispersion slope, the center wavelength, and the bandwidth are $-0.3\left(\mathrm{ps} / \mathrm{nm}^{2}\right) / \mathrm{km}, 1550 \mathrm{~nm}$, and $3 \mathrm{THz}$, respectively. The higherorder dispersion parameters are not considered here. The spectral resolution improves with larger GVD, as expected.

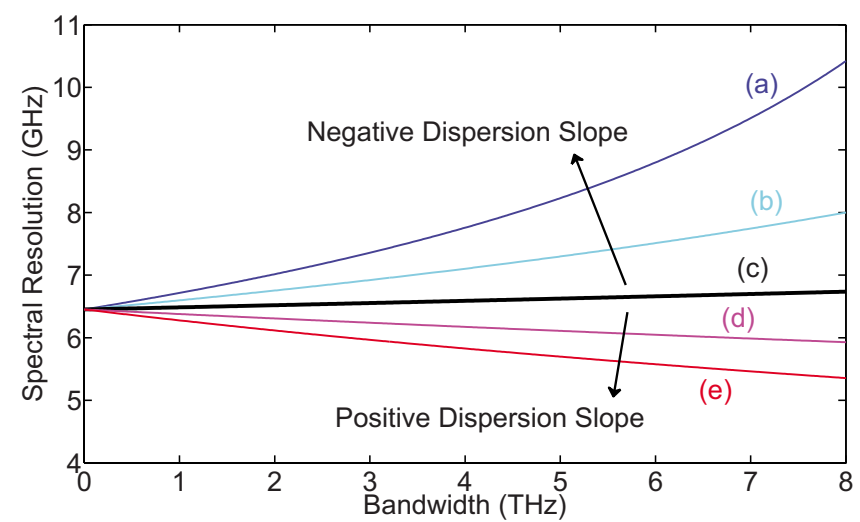

FIG. 7. (Color online) The effect of $\beta_{3}$ and the bandwidth $\Omega_{S}$ $-\omega_{S}$ on the spectral resolution given in Eq. (34) for various values of $d D /\left.d \lambda\right|_{\lambda_{S}}$ (the relation between the GVD coefficients and dispersion parameters is given in Appendix B): (a) $-1\left(\mathrm{ps} / \mathrm{nm}^{2}\right) / \mathrm{km}$, (b) $-0.5\left(\mathrm{ps} / \mathrm{nm}^{2}\right) / \mathrm{km}$, (c) $0\left(\mathrm{ps} / \mathrm{nm}^{2}\right) / \mathrm{km}$, (d) $0.5\left(\mathrm{ps} / \mathrm{nm}^{2}\right) / \mathrm{km}$, and (e) $1\left(\mathrm{ps} / \mathrm{nm}^{2}\right) / \mathrm{km}$. The dispersion length, the center wavelength, and the dispersion parameter $D\left(\lambda_{S}\right)$ used for the figure are $z$ $=50 \mathrm{~km}, 1550 \mathrm{~nm}$, and $-120(\mathrm{ps} / \mathrm{nm}) / \mathrm{km}$, respectively. The higher-order dispersion parameters are not considered here. The effect of $\beta_{3}$ becomes evident with larger bandwidth.

tion system for ADFT measurements consists of a singlepixel high-speed photodetector, analog electronics such as filters and amplifiers, and an analog-to-digital converter or oscilloscope, which ideally would have large real-time bandwidth.

In most cases, the temporal resolution of the digitizer is the limiting factor, as the bandwidths of the state-of-the-art photodetector and digitizer are about 100 and $16 \mathrm{GHz}$, respectively. It is given by the rise time of the digitizer $[17,18]$

$$
\tau_{\text {dig }}=\frac{0.35}{f_{\text {dig }}},
$$

where $f_{\text {dig }}$ is the bandwidth of the digitizer. Figure 8 shows the temporal resolution of the digitizer and the temporal resolution imposed by ADFT. It is ideal to have an ADFT system whose temporal resolution (hence, the spectral resolution of ADFT) is limited by the available dispersion, not the bandwidth of the digitizer. For example, in the case of a typical dispersive fiber with $-120(\mathrm{ps} / \mathrm{nm}) / \mathrm{km}$, it is desirable to have a digitizer bandwidth of $10 \mathrm{GHz}$ or higher not to be limited by the bandwidth of the digitizer for a typical dispersion length of $1-100 \mathrm{~km}$.

The condition for a dispersion-limited ADFT system is given by

$$
\tau_{\text {dig }} \leq \Delta T
$$

where Eq. (35) has been used. This equation can be rewritten as 


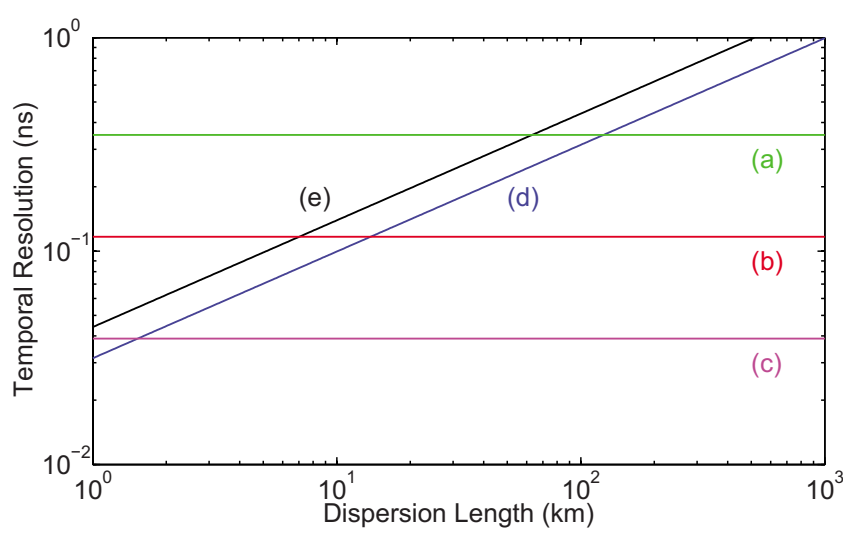

FIG. 8. (Color online) The required temporal resolution of the digitizer or oscilloscope ([(a)-(c)] and the temporal resolution imposed by ADFT [(d)-(e)] for various values of the digitizer bandwidth $f_{\text {dig }}$ and dispersion parameters $\left[D\left(\lambda_{S}\right), d D /\left.d \lambda\right|_{\lambda_{S}}\right.$ : (a) $1 \mathrm{GHz}$, (b) $3 \mathrm{GHz}$, (c) $9 \mathrm{GHz},(\mathrm{d})-60(\mathrm{ps} / \mathrm{nm}) / \mathrm{km}$ and $0.3\left(\mathrm{ps} / \mathrm{nm}^{2}\right) / \mathrm{km}$, and (e) $-120(\mathrm{ps} / \mathrm{nm}) / \mathrm{km}$ and $0.3\left(\mathrm{ps} / \mathrm{nm}^{2}\right) / \mathrm{km}$. The center wavelength and bandwidth are $1550 \mathrm{~nm}$ and $1 \mathrm{THz}$, respectively. It is ideal to have an ADFT system whose temporal resolution is limited by the available dispersion. For the system with (a) and (d), a dispersion length of about $125 \mathrm{~km}$ is required for the dispersive fiber, whereas for the system with (c) and (e), it is an entirely dispersion-limited system, not influenced by the bandwidth of the digitizer for a dispersion length of 1-1000 km.

$$
f_{\text {dig }} \geq \frac{0.35}{2 \pi \sqrt{\sum_{m=0}^{\infty} \frac{\beta_{m+2}}{m !} z\left(\Omega_{S}-\omega_{S}\right)^{m}}},
$$

which is the requirement on the bandwidth of the digitizer and shown in Fig. 9. As the dispersion increases, the requirement for the digitizer bandwidth is relaxed, as expected.

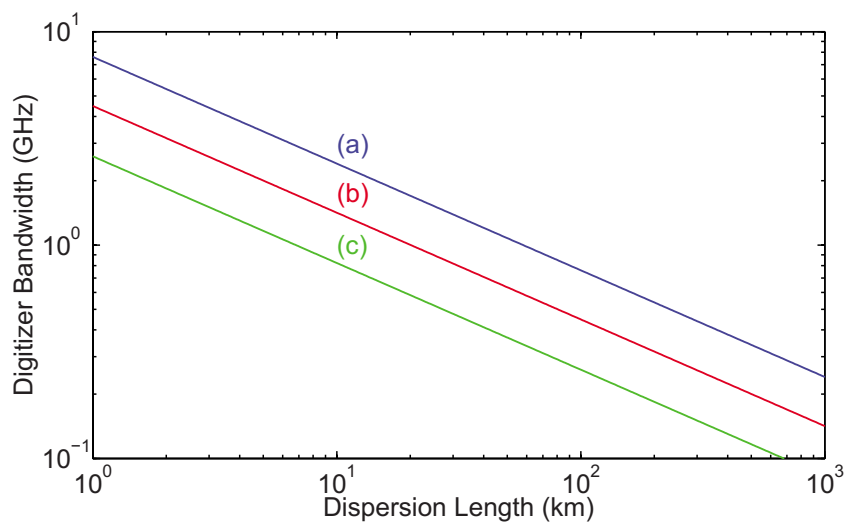

FIG. 9. (Color online) The digitizer bandwidth required for the given dispersion length for various values of the dispersion parameters $\left[D\left(\lambda_{S}\right), d D /\left.d \lambda\right|_{\lambda_{S}}\right]$ (the relation between the GVD coefficients and dispersion parameters is given in Appendix B): (a) $-40(\mathrm{ps} / \mathrm{nm}) / \mathrm{km}$ and $0.3\left(\mathrm{ps} / \mathrm{nm}^{2}\right) / \mathrm{km}, \quad$ (b) $-120(\mathrm{ps} / \mathrm{nm}) / \mathrm{km}$ and $0.3\left(\mathrm{ps} / \mathrm{nm}^{2}\right) / \mathrm{km}$, and (c) $-360(\mathrm{ps} / \mathrm{nm}) / \mathrm{km}$ and $0.3\left(\mathrm{ps} / \mathrm{nm}^{2}\right) / \mathrm{km}$. The center wavelength and bandwidth are 1550 $\mathrm{nm}$ and $1 \mathrm{THz}$, respectively. As the dispersion increases, the requirement for the bandwidth of the digitizer is relaxed.

\section{CONCLUSIONS}

In conclusion, we have presented the theory of ADFT. We have derived the general equation for ADFT from the generalized nonlinear Schrödinger equation with all the higherorder GVD coefficients in the presence of multiple pump fields. We have also shown how insufficient GVD results in incomplete frequency-to-time mapping. In addition, we have also derived the spectral resolution of ADFT as well as its temporal equivalent. We have shown how this value compares with an effective resolution set by the finite bandwidth of the electronic digitizer or oscilloscope used to capture the time-domain representation of the optical spectrum. We have also examined the detailed evolution of the spectrum of an optical pulse into a time-domain waveform and the effect of higher-order GVD coefficients on it. As numerous applications of ADFT are being recognized [1-5], the theory and results presented here are expected to be valuable in design of a new class of spectrometers that are based on the frequency-to-time transformation.

\section{ACKNOWLEDGMENTS}

This work was partially supported by the Defense Advanced Research Projects Agency (DARPA). We are grateful to our colleagues, especially Ali Motafakker-Fard and Shalabh Gupta, at University of California, Los Angeles for valuable discussions.

\section{APPENDIX A: DERIVATION OF THE GENERALIZED NONLINEAR SCHRODINGER EQUATION IN PRESENCE OF MULTIPLE PUMP FIELDS}

In this appendix, starting with Maxwell's wave equation, we derive the generalized nonlinear Schrödinger equation with all the GVD coefficients in the presence of multiple pump fields, and simplify the equation under some assumptions. Throughout this paper, the formalism used to derive and solve the generalized nonlinear Schrödinger equation is similar to that employed in Refs. [14,19,20].

Maxwell's wave equation is given by [21]

$$
\nabla^{2} \mathbf{E}-\frac{1}{c^{2}} \frac{\partial^{2} \mathbf{E}}{\partial t^{2}}=\mu_{0} \frac{\partial^{2} \mathbf{P}}{\partial t^{2}},
$$

where $c$ is the speed of light in vacuum, $\mu_{0}$ is the vacuum permeability, $\mathbf{E}(\mathbf{r}, t)$ is the total electric field, and $\mathbf{P}(\mathbf{r}, t)$ is the total induced polarization, which is the sum of the linear polarization $\mathbf{P}_{L}(\mathbf{r}, t)$, the nonlinear polarization $\mathbf{P}_{N L}(\mathbf{r}, t)$, and the noise-induced polarization $\mathbf{P}_{N}(\mathbf{r}, t)$ such that

$$
\mathbf{P}(\mathbf{r}, t)=\mathbf{P}_{L}(\mathbf{r}, t)+\mathbf{P}_{N L}(\mathbf{r}, t)+\mathbf{P}_{N}(\mathbf{r}, t) .
$$

Throughout this paper, we will ignore the noise-induced polarization $\mathbf{P}_{N}(\mathbf{r}, t)$ and discuss its effect on ADFT in a separate paper [22]. Assuming that the medium (an optical fiber in this case) has no second-order nonlinear polarization, $\mathbf{P}_{N L}(\mathbf{r}, t)$ is approximately the sum of the dominant polarizations caused by the Kerr effect $\mathbf{P}_{K}(\mathbf{r}, t)$ and the Raman effect $\mathbf{P}_{R}(\mathbf{r}, t)$ such that 


$$
\mathbf{P}_{N L}(\mathbf{r}, t) \simeq \mathbf{P}_{K}(\mathbf{r}, t)+\mathbf{P}_{R}(\mathbf{r}, t) .
$$

Thus, the total polarization $\mathbf{P}$ is given by

$$
\begin{aligned}
\mathbf{P}(\mathbf{r}, t)= & \mathbf{P}_{L}(\mathbf{r}, t)+\mathbf{P}_{K}(\mathbf{r}, t)+\mathbf{P}_{R}(\mathbf{r}, t) \\
= & \epsilon_{0} \int_{-\infty}^{\infty} \chi_{L}\left(t-t^{\prime}\right) \mathbf{E}\left(\mathbf{r}, t^{\prime}\right) d t^{\prime} \\
& +\mathbf{E}(\mathbf{r}, t)\left[\chi_{K} \mathbf{E}(\mathbf{r}, t) \cdot \mathbf{E}(\mathbf{r}, t)\right. \\
& \left.+\int_{-\infty}^{\infty} \chi_{R}\left(t-t^{\prime}\right) \mathbf{E}\left(\mathbf{r}, t^{\prime}\right) \cdot \mathbf{E}\left(\mathbf{r}, t^{\prime}\right) d t^{\prime}\right],
\end{aligned}
$$

where $\epsilon_{0}$ is the vacuum permittivity, $\chi_{L}(t)$ is the linear susceptibility, $\chi_{K}$ is the Kerr susceptibility, and $\chi_{R}(t)$ is the Raman susceptibility.

The total electric field $\mathbf{E}(\mathbf{r}, t)$ can be written as the sum of the Stokes and multiple pump fields and are given by

$$
\mathbf{E}(\mathbf{r}, t)=\frac{1}{2} \hat{x}\left[E_{S}(\mathbf{r}, t) e^{-i \omega_{S} t}+\sum_{j=1}^{\infty} E_{p_{j}}(\mathbf{r}, t) e^{-i \omega_{p_{j}} t}+\text { c.c. }\right],
$$

where $\hat{x}$ is the polarization unit vector, $\omega_{S}$ is the carrier frequency of the Stokes field, and $\omega_{p_{j}}$ is the carrier frequency of the pump field $j$. Here c.c. means the complex conjugate. The dot product $\mathbf{E} \cdot \mathbf{E}$ is, then, given by

$$
\begin{aligned}
\mathbf{E} \cdot \mathbf{E}= & \frac{1}{2}\left|E_{S}\right|^{2}+\frac{1}{2} \sum_{j=1}^{\infty}\left|E_{p_{j}}\right|^{2}+\frac{1}{2} \sum_{j=1}^{\infty} E_{p_{j}}^{*} E_{S} e^{i\left(\omega_{p_{j}}-\omega_{S}\right) t} \\
& +\frac{1}{2} \sum_{j=1}^{\infty} E_{p_{j}} E_{S}^{*} e^{-i\left(\omega_{p_{j}}-\omega_{S}\right) t}+\frac{1}{2} \sum_{j=1}^{\infty} \sum_{k=1, k \neq j}^{\infty} E_{p_{j}}^{*} E_{p_{k}} e^{i\left(\omega_{p_{j}}-\omega_{p_{k}}\right) t} \\
& +\frac{1}{2} \sum_{j=1}^{\infty} \sum_{k=1, k \neq j}^{\infty} E_{p_{j}} E_{p_{k}}^{*} e^{-i\left(\omega_{p_{j}}-\omega_{p_{k}}\right) t} \\
& + \text { (terms that require phase-matching). }
\end{aligned}
$$

The first term and the first summation term are the self couplings, the second and third summation terms are the couplings between the Stokes and pump fields, and the fourth and fifth summation terms are the couplings between the pumps. Likewise, the Raman polarization can be written as the sum of the polarizations induced by the Stokes and multiple pump fields and is given by

$$
\mathbf{P}_{R}(\mathbf{r}, t)=\frac{1}{2} \hat{x}\left[P_{R}^{S}(\mathbf{r}, t) e^{-i \omega_{S} t}+\sum_{j=1}^{\infty} P_{R}^{p_{j}}(\mathbf{r}, t) e^{-i \omega_{p_{j}} t}+\text { c.c. }\right] .
$$

Substituting Eq. (A5) into Eq. (A7) using Eq. (A6) and dropping the terms that require phase-matching since they are small compared to the other terms, the Stokes component of the Raman polarization is found to be

$$
\begin{aligned}
P_{R}^{S}(\mathbf{r}, t)= & \frac{1}{4} \epsilon_{0} E_{S}(t) \int_{-\infty}^{\infty} \chi_{R}\left(t-t^{\prime}\right)\left[\left|E_{S}\left(t^{\prime}\right)\right|^{2}+\sum_{j=1}^{\infty}\left|E_{p_{j}}\left(t^{\prime}\right)\right|^{2}\right] d t^{\prime} \\
& +\frac{1}{2} \epsilon_{0} \sum_{j=1}^{\infty} E_{p_{j}}(t) \int_{-\infty}^{\infty} \chi_{R}\left(t-t^{\prime}\right) E_{S}\left(t^{\prime}\right) E_{p_{j}}^{*}\left(t^{\prime}\right) \\
& \times e^{-i\left(\omega_{S}-\omega_{p_{j}}\right)\left(t^{\prime}-t\right)} d t^{\prime},
\end{aligned}
$$

where the pump-pump couplings have been ignored since their Raman response is much weaker than the Raman response of the Stokes field by the pump fields when considering relatively narrow bandwidths $(\sim 40 \mathrm{~nm})$. This approximation is valid since the Raman response function of glass fibers is peaked at $450 \mathrm{~cm}^{-1}$ from the pump frequency [20]. To consider even larger bandwidths, the effect of the pumppump couplings on the Stokes component of the Raman polarization needs to be taken into account. $P_{R}^{p_{j}}$ can also be found similarly.

Before deriving the generalized nonlinear Schrödinger equation from Eq. (A1), we make a few simplifications with some fair assumptions, similar to those used in Refs. $[14,19,20]$. Assuming that nonlinear effects in the fiber do not significantly change the fundamental fiber-mode distribution of the Stokes and pump fields $\left[T_{S}(x, y), T_{p_{j}}(x, y)\right]$ perpendicular to the direction of the field propagation $\hat{z}$, it can be separated out from the amplitude envelope of the fields $\left[A_{S}(z, t), A_{p_{j}}(z, t)\right]$ by using

$$
\begin{gathered}
E_{S}(\mathbf{r}, t)=T_{S}(x, y) A_{S}(z, t) e^{i \beta_{0}^{S} z}, \\
E_{p_{j}}(\mathbf{r}, t)=T_{p_{j}}(x, y) A_{p_{j}}(z, t) e^{i \beta_{0}^{p_{z} z},}
\end{gathered}
$$

where $\beta_{0}^{S}$ and $\beta_{0}^{p_{j}}$ are the wave numbers for the Stokes and pump fields. Assuming that $T_{S}(x, y)$ and $T_{p_{j}}(x, y)$ are approximately equal in a single mode fiber such that

$$
T_{S}(x, y) \simeq T_{p_{j}}(x, y)=T(x, y) .
$$

Furthermore, the field amplitudes $A_{S}(z, t), A_{p_{j}}(z, t)$ can be normalized such that

$$
\begin{aligned}
& u_{S}(z, t)=\kappa_{S} A_{S}(z, t) \sqrt{\int_{-\infty}^{\infty} T^{2}(x, y) d x d y}, \\
& u_{p_{j}}(z, t)=\kappa_{p_{j}} A_{p_{j}}(z, t) \sqrt{\int_{-\infty}^{\infty} T^{2}(x, y) d x d y},
\end{aligned}
$$

where

$$
\kappa_{S}^{2}=\frac{1}{2} n_{S} \sqrt{\frac{\epsilon_{0}}{\mu_{0}}}, \quad \kappa_{p_{j}}^{2}=\frac{1}{2} n_{p_{j}} \sqrt{\frac{\epsilon_{0}}{\mu_{0}}} .
$$

Here $n_{S}$ and $n_{p_{j}}$ are the refractive index of the fiber at the Stokes and pump frequencies. The Kerr susceptibility can be absorbed into the nonlinear coefficient, such that 


$$
\gamma_{S}=\frac{\omega_{S} n_{2}^{S}}{c A_{\mathrm{eff}} \kappa_{S}}, \quad \gamma_{p_{j}}=\frac{\omega_{p_{j}} n_{2}^{p_{j}}}{c A_{\mathrm{eff}} \kappa_{p_{j}}},
$$

where $n_{2}^{S}$ and $n_{2}^{p_{j}}$ are the nonlinear refractive index at the Stokes and pump frequencies and are given by

$$
n_{2}^{S}=\frac{3}{8 n_{S}} \chi_{K}\left(1+\frac{2 \chi_{R}^{0}}{3 \chi_{K}}\right), \quad n_{2}^{p_{j}}=\frac{3}{8 n_{p_{j}}} \chi_{K}\left(1+\frac{2 \chi_{R}^{0}}{3 \chi_{K}}\right) .
$$

Here $\chi_{R}^{0}$ is the normalization coefficient for the Raman response function, such that

$$
\chi_{R}(t)=\chi_{R}^{0} h_{R}(t),
$$

where $h_{R}(t)$ satisfies $\int_{-\infty}^{\infty} h_{R}(t) d t=1$ and $A_{\text {eff }}$ is the effective core area of the fiber and is given by

$$
A_{\mathrm{eff}}=\left(\int_{-\infty}^{\infty} \int_{-\infty}^{\infty} T^{2}(x, y) d x d y\right)^{2} / \int_{-\infty}^{\infty} \int_{-\infty}^{\infty} T^{4}(x, y) d x d y .
$$

In addition, the fraction of the nonlinearity due to molecular vibrations is given by

$$
f_{R}=\left(1+\frac{3 \chi_{K}}{2 \chi_{0}}\right)^{-1}
$$

Now that we have made the simplifications based on several fair assumptions, we are ready to derive the generalized nonlinear Schrödinger equation from Eq. (A1) using the method in Refs. [14,19], where the equation is simplified in the frequency domain which is defined by

$$
u_{S}(z, t)=\frac{1}{2 \pi} \int_{-\infty}^{\infty} \tilde{u}_{S}\left(z, \omega-\omega_{S}\right) e^{-i\left(\omega-\omega_{S}\right) t} d \omega .
$$

Assuming that the nonlinear and noise contributions to $\mathbf{P}_{N L}(\mathbf{r}, t)$ are a small perturbation to the refractive index and the amplitudes $u_{S}, u_{p_{j}}$ are slowly varying functions so that their frequency dependence is negligible, substituting Eqs. (A4) and (A5) with Eq. (A9) into Eq. (A1) yields the coupled equation for the Stokes,

$$
\begin{aligned}
& \frac{\partial u_{S}}{\partial z}-i \sum_{m=1}^{\infty} \frac{i^{m} \beta_{m}}{m !} \frac{\partial^{m} u_{S}}{\partial t^{m}}+\frac{\alpha_{S}}{2} u_{S} \\
& =i \gamma_{S}\left(1-f_{R}\right) u_{S}\left[\left|u_{S}(t)\right|^{2}+2 \sum_{j=1}^{\infty}\left|u_{p_{j}}(t)\right|^{2}\right] \\
& +i \gamma_{S} f_{R} u_{S} \int_{-\infty}^{\infty} h_{R}\left(t-t^{\prime}\right)\left[\left|u_{S}\left(t^{\prime}\right)\right|^{2}+\sum_{j=1}^{\infty}\left|u_{p_{j}}\left(t^{\prime}\right)\right|^{2}\right] d t^{\prime} \\
& +i \gamma_{S} f_{R} \sum_{j=1}^{\infty} u_{p_{j}} \int_{-\infty}^{\infty} h_{R}\left(t-t^{\prime}\right) u_{S}\left(t^{\prime}\right) u_{p_{j}}^{*}\left(t^{\prime}\right) \\
& \quad \times e^{-i\left(\omega_{p_{j}}-\omega_{S}\right)\left(t-t^{\prime}\right)} d t^{\prime}
\end{aligned}
$$

where we have dropped the superscript $s$ for all $\beta_{m}$ for simplicity. The coupled equations for the pump fields can be found similarly. The first term on the left-hand side describes how the Stokes field amplitude evolves with distance $z$. The summation terms on the left hand side are responsible for the effect of dispersion on the Stokes field. The last term on the left hand side describes the optical loss in the fiber for the Stokes field. The terms in the bracket on the right-hand side describe the Kerr-induced contribution to self-phase modulation and cross-phase modulation, respectively. The terms in the first integral on the right-hand side describe the molecular contribution to self-phase modulation and cross-phase modulation, respectively. The final summation terms are responsible for Raman amplification. Equation (1) is valid for Stokes pulse widths up to the limit of the slowly varying envelope approximation $(\sim 10 \mathrm{fs})$.

In general, the nonlinear parameter $\gamma_{S}$ and the loss parameter $\alpha_{S}$ are frequency dependent so that they can be expanded around the center frequency $\omega_{S}$ and expressed in the frequency domain as

$$
\gamma_{S}(\omega)=\gamma_{0}^{S}\left(\omega_{S}\right)+\gamma_{1}^{S}\left(\omega-\omega_{S}\right)+\frac{1}{2} \gamma_{2}^{S}\left(\omega-\omega_{S}\right)^{2}+\cdots,
$$

$$
\alpha_{S}(\omega)=\alpha_{0}^{S}\left(\omega_{S}\right)+\alpha_{1}^{S}\left(\omega-\omega_{S}\right)+\frac{1}{2} \alpha_{2}^{S}\left(\omega-\omega_{S}\right)^{2}+\cdots
$$

However, employing the slowly varying amplitude approximation under the assumption that the Stokes pulse width is more than $100 \mathrm{fs}$, the higher-order terms can be dropped. In the main text, we use only the leading terms so that $\gamma_{S}=\gamma_{0}^{S}$ and $\alpha_{S}=\alpha_{0}^{S}$, where the superscript $S$ will be dropped for simplicity.

\section{APPENDIX B: WAVELENGTH-TIME RELATION IN TERMS OF THE DISPERSION PARAMETERS}

In this paper, we have demonstrated the wavelength-time relation of the generalized ADFT in terms of the GVD coefficients $\beta_{m}$. However, it is more useful to express it in terms of the dispersion parameters such as the zeroth-order dispersion parameter $D$ and the first-order dispersion parameter or the so-called dispersion slope $d D / d \lambda$, where $\lambda$ is the wavelength, than the GVD coefficients, as the dispersion parameters are often used to describe the dispersive properties of optical fibers as a function of the wavelength rather than the frequency. In this appendix, we express the generalized wavelength-time relation in terms of the dispersion parameters.

The dispersion parameter $D$ is defined by [20]

$$
D=\frac{d}{d \lambda}\left(\frac{d \beta}{d \omega}\right)=-\frac{2 \pi c}{\lambda^{2}} \frac{d^{2} \beta}{d \omega^{2}},
$$

from which $D, d D / d \lambda$, and $d^{2} D / d \lambda^{2}$ evaluated at the center frequency $\lambda_{S}=2 \pi c / \omega_{S}$ are found to be

$$
D\left(\lambda_{S}\right)=\left(-\frac{2 \pi c}{\lambda_{S}^{2}}\right) \beta_{2}
$$




$$
\begin{gathered}
\left.\frac{d D}{d \lambda}\right|_{\lambda_{S}}=-\frac{2}{\lambda_{S}}\left(-\frac{2 \pi c}{\lambda_{S}^{2}}\right) \beta_{2}+\left(-\frac{2 \pi c}{\lambda_{S}^{2}}\right)^{2} \beta_{3}, \\
\left.\frac{d^{2} D}{d \lambda^{2}}\right|_{\lambda_{S}}=\frac{6}{\lambda_{S}^{2}}\left(-\frac{2 \pi c}{\lambda_{S}^{2}}\right) \beta_{2}-\frac{6}{\lambda_{S}}\left(-\frac{2 \pi c}{\lambda_{S}^{2}}\right)^{2} \beta_{3}+\left(-\frac{2 \pi c}{\lambda_{S}^{2}}\right)^{3} \beta_{4} .
\end{gathered}
$$

Equation (B1) can be Taylor expanded around the center wavelength $\lambda=\lambda_{S}$ into

$$
D=\left.\sum_{m=0}^{\infty} \frac{d^{m} D}{d \lambda^{m}}\right|_{\lambda_{S}} \frac{\left(\lambda-\lambda_{S}\right)^{m}}{m !} .
$$

From these equations, $\beta_{S}, \beta_{3}$, and $\beta_{4}$ can be found to be

$$
\beta_{2}=\left(-\frac{\lambda_{S}^{2}}{2 \pi c}\right) D\left(\lambda_{S}\right)
$$

$$
\begin{gathered}
\beta_{3}=\left(-\frac{\lambda_{S}^{2}}{2 \pi c}\right)^{2}\left(\left.\frac{d D}{d \lambda}\right|_{\lambda_{S}}+\frac{2}{\lambda_{S}} D\left(\lambda_{S}\right)\right), \\
\beta_{4}=\left(-\frac{\lambda_{S}^{2}}{2 \pi c}\right)^{3}\left[\left.\frac{d^{2} D}{d \lambda^{2}}\right|_{\lambda_{S}}+\left.\frac{6}{\lambda_{S}} \frac{d D}{d \lambda}\right|_{\lambda_{S}}+\frac{6}{\lambda_{S}^{2}} D\left(\lambda_{S}\right)\right]
\end{gathered}
$$

Substituting these into Eq. (33) and using the Taylor expansion

$$
\Delta \Lambda_{S}=\Lambda_{S}-\lambda_{S}=\sum_{m=1}^{\infty} \frac{1}{m !} \frac{d^{m} \Lambda_{S}}{d \Omega_{S}^{m}}\left(\Delta \Omega_{S}\right)^{m},
$$

where $\Delta \Omega_{S}=\Omega_{S}-\omega_{S}$, we find the wavelength-time relation

$$
T=\left.\sum_{m=1}^{\infty} \frac{z}{m !} \frac{d^{m-1} D}{d \lambda^{m-1}}\right|_{\lambda_{S}}\left(\Lambda_{S}-\lambda_{S}\right)^{m},
$$

which agrees with the result in Ref. [23].
[1] K. Goda, K. K. Tsia, and B. Jalali, Nature (London) 458, 1145 (2009).

[2] D. R. Solli, J. Chou, and B. Jalali, Nat. Photonics 2, 48 (2008).

[3] K. Goda, D. R. Solli, and B. Jalali, Appl. Phys. Lett. 93, 031106 (2008).

[4] K. Goda, K. K. Tsia, and B. Jalali, Appl. Phys. Lett. 93, 131109 (2008).

[5] J. Chou, D. Solli, and B. Jalali, Appl. Phys. Lett. 92, 111102 (2008).

[6] J. Chou, O. Boyraz, D. Sollli, and B. Jalali, Appl. Phys. Lett. 91, 161105 (2007).

[7] J. Chou, Y. Han, and B. Jalali, IEEE Photon. Technol. Lett. 16, 1140 (2004).

[8] J. Hult, R. S. Watt, and C. F. Kaminski, Opt. Express 15, 11385 (2007).

[9] S. Moon and D. Y. Kim, Opt. Express 14, 11575 (2006).

[10] S. Gupta and B. Jalali, Appl. Phys. Lett. 94, 041105 (2009).

[11] D. R. Solli, C. Ropers, and B. Jalali, Phys. Rev. Lett. 101, 233902 (2008).

[12] D. R. Solli, C. Ropers, P. Koonath, and B. Jalali, Nature (London) 450, 1054 (2007)

[13] M. N. Islam, IEEE J. Sel. Top. Quantum Electron. 8, 548
(2002).

[14] C. Headley III and G. P. Agrawal, J. Opt. Soc. Am. B 13, 2170 (1996).

[15] K. S. A. Mori, H. Masuda, and M. Shimizu, J. Lightwave Technol. 21, 1300 (2003).

[16] H. Masuda, A. Mori, K. Shikano, and M. Shimizu, J. Lightwave Technol. 24, 504 (2006).

[17] P. Horowitz and W. Hill, The Art of Electronics (Cambridge University Press, Cambridge, England, 1989).

[18] D. A. Neamen, Electronic Circuit Analysis and Design, 2nd edition (McGraw Hill, New York, 2000).

[19] C. Headley III and G. P. Agrawal, IEEE J. Quantum Electron. 31, 2058 (1995).

[20] G. P. Agrawal, Nonlinear Fiber Optics (Academic Press, New York, 2007).

[21] J. D. Jackson, Classical Electrodynamics (Wiley, New York, 1999).

[22] K. Goda, D. R. Solli, K. K. Tsia, and B. Jalali (to be published).

[23] P. V. Kelkar, F. Coppinger, A. S. Bhushan, and B. Jalali, Electron. Lett. 35, 1661 (1999). 